\title{
El panorama de los ensayos clínicos sobre COVID-19 en América Latina y el Caribe: evaluación y desafíos*
}

\author{
Sarah Carracedo ${ }^{1}$, Ana Palmero ${ }^{1}$, Marcie Neil' , Anisa Hasan-Granier ${ }^{1}$, Carla Saenz ${ }^{1}$ y Ludovic Reveiz ${ }^{1}$
}

Forma de citar

Carracedo S, Palmero A, Neil M, Hasan-Granier A, Saenz C y Reveiz L. el panorama de los ensayos clínicos sobre COVID-19 en América Latina y el Caribe: evaluación y desafíos. Rev Panam Salud Publica. 2021;45:e33.

https://doi.org/10.26633/RPSP.2021.33

RESUMEN

Se está llevando a cabo un número considerable de ensayos clínicos en todo el mundo en respuesta a la pandemia de COVID-19, incluso en países de ingresos bajos y medios como los de América Latina y el Caribe. Sin embargo, la abundancia de estudios no necesariamente acorta el camino para encontrar intervenciones seguras y eficaces frente a la COVID-19. Se analizaron los ensayos para el tratamiento y la prevención de la COVID-19 de los países de América Latina y el Caribe que están registrados en la Plataforma de Registros Internacionales de Ensayos Clínicos de la Organización Mundial de la Salud, y se identificó una tendencia hacia la realización de estudios pequeños, repetitivos y no rigurosos que duplican los esfuerzos y merman recursos limitados sin producir conclusiones significativas sobre la seguridad y la eficacia de las intervenciones evaluadas. Se evaluaron asimismo los desafíos que plantea la realización de investigaciones científicamente sólidas y socialmente valiosas en América Latina y el Caribe a fin de brindar recomendaciones que alienten la realización de ensayos clínicos que tengan más probabilidades de producir evidencia sólida durante la pandemia.

Palabras clave Infecciones por coronavirus; ensayo clínico; ética en investigación; políticas públicas de salud; América Latina; Región del Caribe.

Durante la pandemia de SARS-CoV-2, la urgencia de realizar investigaciones para encontrar tratamientos y vacunas seguros y eficaces ha llevado a una proliferación considerable de ensayos clínicos sobre COVID-19. La rápida reacción de la comunidad científica para promover investigaciones durante este brote ha resultado en la conducción de múltiples ensayos clínicos en varios países de todo el mundo, incluso en países de ingresos bajos y medios como los de América Latina y el Caribe (ALC) (1). Debido al creciente número de casos y muertes por COVID-19 reportados en ALC, la investigación se ha convertido en un esfuerzo esencial para dar respuesta a la pandemia. Sin embargo, la cantidad de estudios que se están realizando en ALC en tan corto tiempo y los retos que presenta el registro de

\footnotetext{
* Traducción no oficial revisada por los autores al español del artículo original en inglés efectuada por la Organización Panamericana de la Salud. En caso de discrepancia, prevalecerá la versión original en inglés. Acceso al artículo original: https://doi.org/10.26633/RPSP.2020.177
}

ensayos clínicos para la Región han dificultado la comprensión y la evaluación de las investigaciones en curso. Este artículo presenta una descripción general y un análisis de los ensayos clínicos para el tratamiento y la prevención de la COVID-19 en ALC, con el fin de evaluar los desafíos y ofrecer recomendaciones para garantizar que estos esfuerzos de investigación puedan generar evidencia significativa.

\section{MATERIALES Y MÉTODOS}

Se realizó una búsqueda de los ensayos clínicos sobre COVID-19 de países de ALC registrados en la Plataforma Internacional de Registros de Ensayos Clínicos (ICTRP por sus siglas

\footnotetext{
1 Organización Panamericana de la Salud, Washington, D.C., Estados Unidos de América. $\bowtie$ Carla Saenz, saenzcar@paho.org
} 
en inglés) de la Organización Mundial de la Salud (OMS) desde el inicio de la pandemia hasta el mes de agosto de 2020. Si bien el objetivo principal de la ICTRP es el registro de ensayos clínicos, en ella también se registran estudios observacionales y protocolos de acceso expandido. Además, esta plataforma no incluye todos los ensayos clínicos que se llevan a cabo en ALC ya que en algunos países no es un requisito obligatorio incluir los estudios en registros que formen parte de la ICTRP. En la Región, solo tres países (Brasil, Cuba y Perú) tienen registros primarios de ensayos clínicos que proporcionan datos a esta plataforma (2). A pesar de estas limitaciones, la ICTRP ofrece el panorama más preciso de la investigación realizada en ALC.

\section{RESULTADOS}

Se realizó una búsqueda de los 47 países y territorios de ALC entre los 5213 ensayos clínicos sobre COVID-19 registrados en el mundo, y se identificaron 285 estudios en la Región: 79 están registrados como estudios observacionales y 206 como estudios de intervención. Dos de estas entradas de la ICTRP se refieren explícitamente a intervenciones llevadas a cabo en el contexto de un uso compasivo o programas de acceso expandido. De los estudios de intervención registrados, 170 evalúan productos para el tratamiento y la prevención. La siguiente descripción se basa en este último subconjunto de ensayos clínicos.

Brasil, México y Argentina (en ese orden), registran el mayor número de ensayos clínicos sobre COVID-19. En conjunto, estos tres países suman el 75,8\% del total de ensayos clínicos para el tratamiento y la prevención registrados de la Región (cuadro 1). De los ensayos clínicos registrados en ALC, 18,2\% son multinacionales e involucran colaboraciones con países ubicados fuera de la Región. No se encontró ninguna iniciativa de investigación colaborativa regional. Dos estudios registrados corresponden a esfuerzos de investigación mundiales; uno de ellos es el ensayo clínico Solidaridad de la OMS que se está llevando a cabo en cinco países de ALC. Por otro lado, la mayoría de los estudios multinacionales está patrocinada por la industria farmacéutica. En cambio, entre los ensayos clínicos que se realizan únicamente en un país, el 78,4\% tiene patrocinadores no comerciales.

Se identificaron 141 ensayos clínicos aleatorizados. De ellos, 77 tienen grupo control y enmascaramiento (al menos parcial), y representan el 45,3\% del total de ensayos clínicos registrados. Respecto al tamaño muestral, 104 ensayos clínicos (61,2\%) tienen una muestra de 200 o menos participantes. El tamaño muestral más pequeño de los ensayos clínicos es de nueve participantes y el mayor es de 115000 participantes, correspondiente a un ensayo clínico de una vacuna.

El 87,6\% de los ensayos clínicos se enfocan en el tratamiento de la COVID-19 y no en intervenciones preventivas. Además, la gran mayoría de las intervenciones estudiadas en la Región corresponde a fármacos con otros usos clínicos aprobados. La administración de plasma de convaleciente, antimaláricos y antivirales son las intervenciones más estudiadas (figura 1). En total se están evaluando en ALC 98 tratamientos y vacunas potenciales: 6 terapias combinadas, 84 monoterapias y 8 vacunas ( 2 con una indicación aprobada para otra enfermedad y 6 contra el SARS-CoV-2). Entre las 98 intervenciones en investigación, tres son nuevas terapias y una vacuna contra la COVID-19 que se están evaluando exclusivamente en Cuba (CIGB 300, CIGB-2020, péptido CIGB-258 y FINLAY-FR-1, respectivamente). Al igual que en el resto del mundo, el plasma
CUADRO 1. Número de ensayos clínicos sobre COVID-19 registrados en OMS/ICTRP en países de ALC, marzo - agosto 19, 2020

\begin{tabular}{|c|c|}
\hline País & $\#$ \\
\hline Brasil & 71 \\
\hline México & 52 \\
\hline Argentina & 24 \\
\hline Colombia & 15 \\
\hline Cuba & 12 \\
\hline Perú & 12 \\
\hline Chile & 9 \\
\hline Ecuador & 2 \\
\hline Puerto Rico & 3 \\
\hline Honduras & 1 \\
\hline República Dominicana & 1 \\
\hline Total & 202 \\
\hline
\end{tabular}

OMS/ICTRP, Plataforma Internacional de Registros de Ensayos Clínicos de la Organización Mundial de la Salud (por sus siglas en inglés)

de convaleciente, la hidroxicloroquina/cloroquina (HCQ/CQ) y la azitromicina son las tres intervenciones más estudiadas en ALC (3).

Muchos países están estudiando las mismas intervenciones en una multiplicidad de estudios. Por ejemplo, se encontraron 27 ensayos clínicos llevados a cabo en un único país que están estudiando el plasma de convaleciente en la Región, y la mayoría de ellos tiene muestras pequeñas $(<100)$. En México, por ejemplo, en siete estudios sobre plasma de convaleciente se enrolaron a 281 participantes. En cuanto a la HCQ/CQ (administrada sola o junto con otro fármaco) hay 26 estudios, de los cuales 14 se están realizando en Brasil. Con respecto al estado de reclutamiento, el 70,3\% de los ensayos clínicos sobre HCQ/ CQ estaba activo a la fecha de la búsqueda.

En cuanto a la población, la mayoría de los ensayos clínicos incluye únicamente a adultos (mayores de 18 años). Los niños pueden participar en 11 ensayos clínicos, con una edad mínima de un mes como criterio de inclusión. Solo un estudio registrado es específico sobre la inclusión de mujeres embarazadas, algo que es incierto en 44 de los ensayos clínicos. En el resto de los estudios $(73,5 \%)$ se excluye a las mujeres embarazadas. Hasta agosto de 2020, ninguno de los ensayos clínicos sobre COVID-19 registrados en ALC ha completado el campo de "resultados" de la ICTRP.

\section{DISCUSIÓN}

Del total de ensayos clínicos realizados a nivel mundial para el tratamiento y la prevención de la COVID-19, una cantidad considerable $(10,2 \%)$ se está llevando a cabo en ALC según el registro en la ICTRP. En cuanto al diseño, un número significativo de los ensayos clínicos identificados tiene tamaños de muestra muy pequeños y carece de grupo de control y, por tanto, no pueden producir evidencia sólida sobre la seguridad y eficacia de las intervenciones que se están evaluando. Las lecciones aprendidas durante epidemias anteriores han demostrado que los esfuerzos de investigación deben centrarse en ensayos clínicos controlados aleatorizados bien diseñados con un gran número de participantes para producir evidencia sólida rápidamente $(4,5)$. Los diseños que impiden a los 
FIGURA 1. Número de ensayos clínicos de COVID-19 por tipo de intervención registrada en la OMS/ICTRP, marzo-19 de agosto del 2020

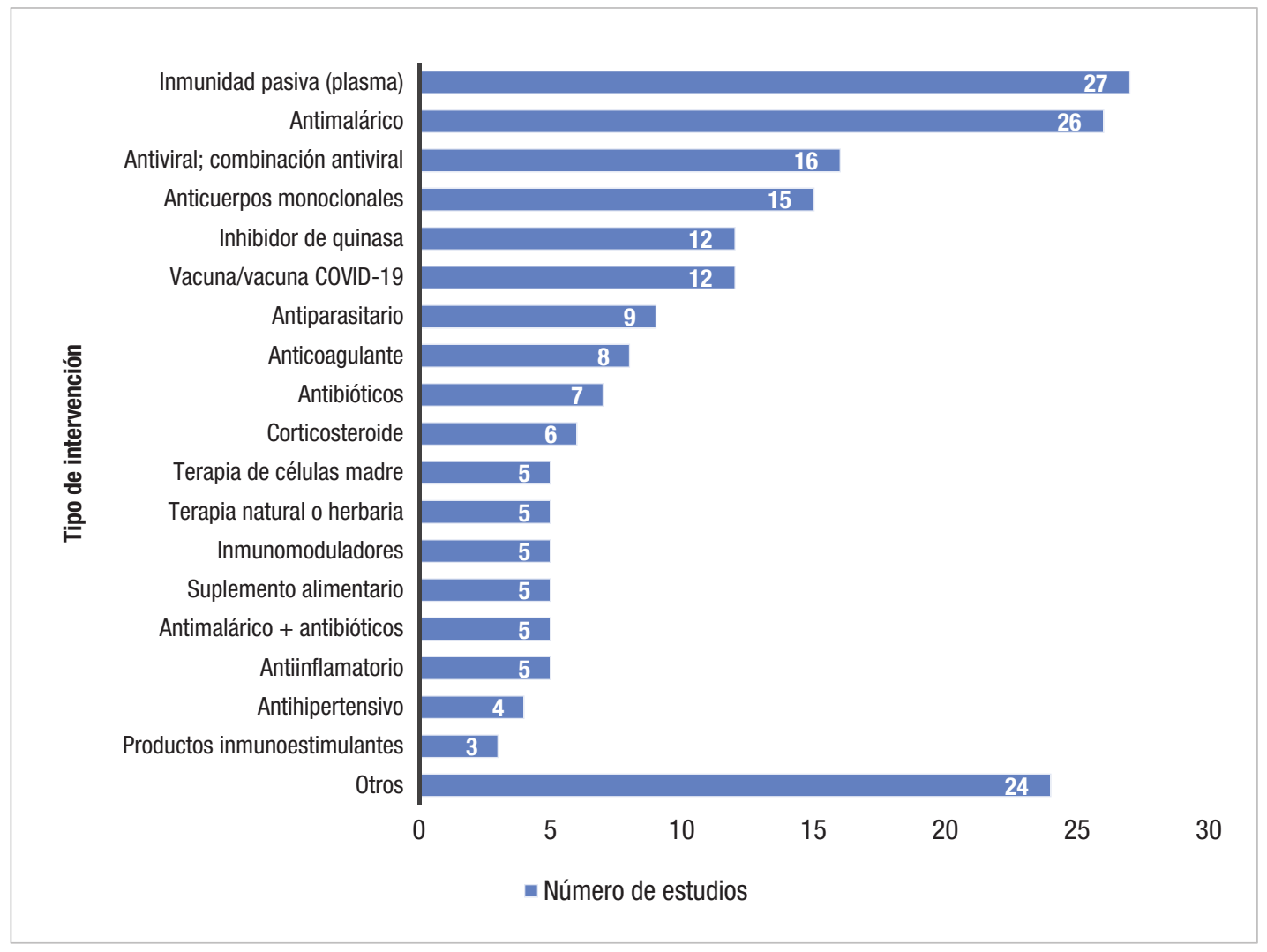

ensayos clínicos responder a su pregunta científica comprometen su validez y son éticamente cuestionables porque los participantes son expuestos a riesgos sin la perspectiva de obtener beneficios como resultado (es decir, conocimiento valioso). Mientras se realicen estudios pequeños y no controlados en la Región, los esfuerzos de investigación no estarán orientados a generar el conocimiento necesario para enfrentar la pandemia.

Asimismo, en ALC los ensayos clínicos a menudo evalúan las mismas intervenciones, lo que da lugar a una gran cantidad de estudios pequeños y repetitivos. También se observa una falta de estudios multicéntricos nacionales y regionales, lo que podría evidenciar debilidades en la coordinación y colaboración en ambos niveles. La falta de experiencia en el diseño y la implementación de grandes ensayos clínicos multicéntricos en la Región, así como las limitaciones de infraestructura para la investigación y el contexto desafiante que plantea la pandemia, podrían representar una explicación adicional. Sin mecanismos efectivos de coordinación y colaboración, los investigadores no logran formar equipos ni intercambiar datos de investigación. La ausencia de coordinación también conduce a la recolección de conjuntos de datos muy diversos, lo que dificulta las comparaciones entre las posibles intervenciones en investigación. Cuando ocurre al interior de una misma jurisdicción, esta ausencia de coordinación puede generar competencia por el reclutamiento de participantes para estudios similares, lo que demora alcanzar el tamaño muestral de los estudios.
Para promover la coordinación y evitar la duplicación de esfuerzos de investigación, los investigadores deben poder conocer los estudios que ya se están realizando. Para esto es indispensable un registro adecuado de ensayos clínicos que sea completo, centralizado y públicamente accesible. Los países deben exigir la inclusión de todos los ensayos clínicos en registros que son parte de la ICTRP. Actualmente, algunos países de la Región carecen de un mecanismo para registrar los ensayos clínicos, y algunos tienen registros nacionales que no están disponibles públicamente o que no forman parte de la ICTRP (6). De igual forma, es fundamental que la ICTRP funcione de manera óptima. Al momento de realizar este estudio, como consecuencia del intenso tráfico de datos en internet durante la pandemia, la herramienta de búsqueda de la plataforma no funcionaba y solo se podía descargar del sitio web un archivo de base de datos de los ensayos clínicos sobre COVID-19 registrados a nivel mundial. Otros desafíos enfrentados fueron los registros de ensayos clínicos con información incompleta y los estudios mal categorizados.

Sin embargo, se puede argumentar que, para promover una rápida colaboración entre equipos de investigación en el contexto de la pandemia, ni siquiera el registro adecuado de ensayos clínicos es suficiente. Cuando se registran los estudios, los protocolos ya han sido elaborados, obtuvieron la aprobación ética y están listos para comenzar con el reclutamiento de participantes. Por ello, se hace necesario contar con mecanismos para que los investigadores compartan sus planes en una 
etapa previa, y así facilitar un trabajo colaborativo que lleve a la articulación de ensayos clínicos multicéntricos. Diseñar e implementar tales mecanismos es un desafío para mejorar nuestra respuesta a la pandemia y a futuras emergencias.

En relación con las intervenciones en estudio, los ensayos clínicos de ALC persisten en evaluar principalmente intervenciones que han demostrado ser potencialmente nocivas, tener una tasa de éxito baja o ser ineficaces, como las relacionadas con HCQ/CQ, hidroxicloroquina y azitromicina, o lopinavir/ ritonavir (7-9). El rápido desarrollo de evidencia científica durante la pandemia plantea la necesidad de supervisar los ensayos clínicos que están en marcha para garantizar que sigan teniendo valor social y estén justificados tanto científicamente como a la luz de su perfil de riesgo-beneficio. Los países de ALC deben fortalecer los mecanismos de supervisión de los ensayos clínicos sobre COVID-19 y tomar las acciones que sean necesarias, como la suspensión y modificación de los estudios que ya no están justificados o carecen de un perfil de riesgo-beneficio favorable y que, por lo tanto, dejan de ser éticos. La implementación de esta supervisión evitará que los participantes estén expuestos a riesgos innecesarios y se utilicen recursos valiosos en investigaciones inútiles. Por otro lado, la escasez de estudios sobre nuevas intervenciones en la Región sugiere la necesidad de mejorar la capacidad en ciencias básicas, la realización de ensayos clínicos locales y el fomento de la innovación.

En cuanto a la población de estudio, los ensayos clínicos de ALC analizados tienden a excluir sistemáticamente a niños y adolescentes, así como a mujeres embarazadas y en período de lactancia (10). Por ejemplo, el lopinavir/ritonavir y la hidroxicloroquina ya son utilizados en mujeres embarazadas con infección por VIH y malaria, respectivamente; el remdesivir se ha probado en mujeres embarazadas durante el brote de Ébola, y las mujeres con esclerosis múltiple reciben interferón beta durante el embarazo (11-13). A pesar de ello, las mujeres embarazadas son excluidas de los ensayos clínicos que estudian intervenciones con estos fármacos. La infección por el SARS-CoV-2 puede afectar a cualquier persona, y la exclusión generalizada de estos grupos sin una justificación adecuada los priva injustamente de los posibles beneficios de la investigación.

A pesar de verse muy afectada durante la pandemia, la gran cantidad de investigación que se está llevando a cabo en la Región como parte de la respuesta a la COVID-19 revela un aumento de las capacidades en investigación y evidencia los desafíos pendientes. Aún se requieren esfuerzos para maximizar la capacidad de ALC para realizar ensayos clínicos que produzcan evidencia sólida y aprovechen al máximo los recursos escasos. Se deben priorizar los ensayos clínicos bien diseñados, de gran escala, multinacionales, aleatorizados y controlados que evalúen intervenciones con resultados prometedores, basados en evidencia actualizada y que incluyan a los participantes de manera equitativa. Para ello es indispensable la coordinación y colaboración temprana entre los múltiples equipos de investigación. Los ensayos clínicos adaptativos con múltiples brazos que investigan diferentes intervenciones simultáneamente se consideran la mejor opción durante la pandemia para producir resultados rápidos entre una variedad de opciones de tratamiento de acuerdo con criterios de valoración y resultados armonizados (14-16). En ese sentido, debería fomentarse una mayor participación de los países de
ALC en iniciativas mundiales de este tipo, como el Ensayo Clínico Solidaridad de la OMS, y el desarrollo de colaboraciones de investigación regionales similares, con miras a fortalecer aún más la capacidad local.

Por último, los desafíos planteados por la pandemia de la COVID-19 exponen la urgencia de fortalecer los sistemas nacionales de investigación y los sistemas nacionales de ética de la investigación dentro de ellos que tienen como objetivo asegurar que la investigación sea efectiva y cumpla los más altos estándares éticos para informar la toma de decisiones durante y después de la pandemia. Los esfuerzos en curso de la Organización Panamericana de la Salud para fortalecer las capacidades de ALC en la realización de investigación ética y rigurosa deben intensificarse para fomentar redes de investigación colaborativa en toda la Región que sean capaces de catalizar investigaciones efectivas y de alto impacto. Contar con sistemas nacionales de investigación y redes de colaboración bien establecidos permitirá que los países y territorios de ALC estén mejor preparados en el futuro para dar respuesta a emergencias de salud mediante la investigación.

\section{CONCLUSIÓN}

La evaluación de los ensayos clínicos llevados a cabo en la Región revela una tendencia hacia la realización de estudios pequeños, repetitivos y no rigurosos, que duplican esfuerzos y desaprovechan los recursos limitados sin proporcionar evidencia sólida sobre la seguridad y eficacia de las intervenciones que se están probando. Los países de ALC necesitan aunar esfuerzos y dirigir sus recursos hacia intervenciones en investigación con una mayor tasa de éxito. Los ensayos clínicos controlados, aleatorizados, de alta calidad y bien diseñados con muestras grandes y criterios de inclusión equitativos están mejor posicionados para contribuir de manera significativa al descubrimiento de terapias y vacunas seguras y eficaces para la COVID-19. La existencia de mecanismos de coordinación y colaboración para ensayos clínicos en y entre los países de la Región, el registro de ensayos clínicos en registros públicos y globales que formen parte de la ICTRP, una mayor participación en iniciativas globales de investigación, y el fortalecimiento de los sistemas nacionales de investigación son elementos clave para mejorar nuestra respuesta de investigación regional a la pandemia de la COVID-19 y más allá de ella.

Contribución de los autores. Concepción y diseño: AP, SC, NM, AHG, CS, LR; búsqueda de literatura: AP, SC, CS, LR; recolección de datos: AP, SC, NM, AHG; análisis e interpretación de datos: AP, SC, NM, AHG, CS, LR; elaboración y revisión del manuscrito: AP, SC, NM, CS, LR; aprobación final del manuscrito: AP, SC, NM, AHG, CS, LR. SC y AP contribuyeron por igual y son primeras autoras del artículo.

Conflictos de intereses. Ninguno declarado por los autores.

Financiación. El estudio fue parcialmente financiado por el Wellcome Trust, grant 220028/Z/19/Z.

Declaración. Las opiniones expresadas en este manuscrito son responsabilidad de los autores y no reflejan necesariamente los criterios ni la política de la RPSP/PAJPH o de la OPS. 


\section{REFERENCIAS}

1. Organización Mundial de la Salud. A Coordinated Global Research Roadmap: 2019 Novel Coronavirus. R \& D Blueprint. [Internet] 12 de marzo 2020 [citadoel27 Ago 2020]. Disponibleen:https:/ / www.who. int/publications/m/item/a-coordinated-global-research-roadmap

2. Organización Mundial de la Salud. Plataforma de registros internacionales de ensayos clínicos [Internet]. Ginebra: OMS; 2020 [actualizado el 26 Ago 2020; citado el 28 Ago 2020]. Disponible en: https://www.who.int/ictrp/network/primary/es/

3. Rafel JB, Marin M, Heaney T. Covid-19 clinical trials explorer [Internet]. 24 Abr 2020 [actualizado el 19 Junio 2020; citado el 27 Ago 2020]. Disponibleen:https://public.tableau.com/profile/marinamarin\#!/ vizhome/covidTrials/COVID-19ClinicalTrialsExplorer

4. London AJ, Kimmelman J. Against pandemic research exceptionalism. Science. 2020;368(6490):476-7. doi: 10.1126/science.abc1731.

5. Lane HC, Fauci AS. Research in the Context of a Pandemic [publicado en línea antes de la impresión, 17 Jul 2020]. NEJM. doi:10.1056/ NEJMe2024638.

6. García-Vello P, Smith E, Elias V, Florez Pinzon C, Reveiz L. Adherence to clinical trial registration in countries of Latin America and the Caribbean, 2015. Rev Panam Salud Publica. 2018;42:e44. doi. org/10.26633/RPSP.2018.44

7. Universidad de Oxford. RECOVERY Trial [Internet]. [citado el 31 Ago 2020]. Disponible en: https:/ / www.recoverytrial.net/

8. Institutos Nacionales de Salud de los Estados Unidos de América. What's new in the Guidelines [Internet] [actualizado el 3 Nov 2020; citado el 31 Ago 2020]. Disponible en: https://www.covid19treatmentguidelines.nih.gov/whats-new/

9. Organización Panamericana de la Salud. Ongoing living update of potential COVID-19 therapeutics: summary of rapid systematic reviews [Internet] [actualizado el 16 Jun 2020; citado el 27 Ago 2020]. Disponible en: https://iris.paho.org/bitstream/ handle/10665.2/52294/PAHOIMSEIHCOVID-19200008_eng. pdf? sequence $=1 \&$ isAllowed $=y$

10. Hwang TJ, Randolph AG, Bourgeois FT. Inclusion of Children in Clinical Trials of Treatments for Coronavirus Disease 2019 (COVID19). JAMA Pediatr. Publicado en línea el 7 May 2020. doi:10.1001/ jamapediatrics.2020.1888s

11. ClinicalInfo.hiv.gov. Lopinavir/Ritonavir (Kaletra, LPV/r): Recommendations for the Use of Antiretroviral Drugs in Pregnant
Women with HIV Infection and Interventions to Reduce Perinatal HIV Transmission in the United States. Office of AIDS Research. [Internet] [actualizado el 24 Dec 2019; citado el 27 Ago 2020]. Disponible en: https://clinicalinfo.hiv.gov/en/guidelines/perinatal/ lopinavirritonavir-kaletra-lpvr

12. Mulangu S, Dodd LE, Davey RT, Tshiani Mbaya O, Proschan M, Mukadi D, et al. A Randomized, Controlled Trial of Ebola Virus Disease Therapeutics. NEJM. 2020;381(24):2293-2303. doi: 10.1056/ NEJMoa1910993.

13. Hellwig K, Geissbuehler Y, Sabidó M, Popescu C, Adamo A, Klinger J, et al. Pregnancy outcomes in interferon-beta-exposed patients with multiple sclerosis: results from the European Interferon-beta Pregnancy Registry. J Neurol. 2020;267(6):1715-23. doi: 10.1007/ s00415-020-09762

14. Eichler HG, Cavaleri M, Enzmann H, Scotti F, Sepodes B, Sweeney F, Vamvakas S, Rasi G. Clinical trials for Covid-19: can we better use the short window of opportunity? Clin Pharmacol Ther 2020;108(4):730-3. doi:10.1002/cpt.1891

15. Agencia Europea de Medicamentos, Comité de Medicamentos de Uso Humano (CHMP). Call to pool research resources into large multi-centre, multi-arm clinical trials to generate sound evidence on COVID-19 treatments. [En prensa] 19 Marzo 2020. [citado el 27 Ago 2020]. Disponible en: https://www.ema.europa.eu/en/ documents / press-release / call-pool-research-resources-largemulti-centre-multi-arm-clinical-trials-generate-sound-evidence en.pdf

16. Organización Panamericana de la Salud. Consideraciones para la supervisión regulatoria de los ensayos clínicos en la pandemia de COVID-19. Washington D.C.: OPS; 2020. Disponible en: https:// www.paho.org/en/documents/considerations-regulatory-oversight-clinical-trials-covid-19-pandemic

Manuscrito (original en inglés) recibido el 15 de octubre de 2020; versión revisada aceptada para publicación el 19 de noviembre de 2020.

\section{The landscape of COVID-19 clinical trials in Latin America and the Caribbean: assessment and challenges}

ABSTRACT A considerable number of clinical trials is being conducted globally in response to the COVID-19 pandemic, including in low- and middle-income countries such as those in the Latin America and Caribbean region (LAC). Yet, an abundance of studies does not necessarily shorten the path to find safe and efficacious interventions for COVID-19. We analyze the trials for COVID-19 treatment and prevention that are registered from LAC countries in the International Clinical Trials Registry Platform, and identify a trend towards small repetitive non-rigorous studies that duplicate efforts and drain limited resources without producing meaningful conclusions on the safety and efficacy of the interventions being tested. We further assess the challenges to conducting scientifically sound and socially valuable research in the LAC region in order to inform recommendations to encourage clinical trials that are most likely to produce robust evidence during the pandemic.

Keywords Coronavirus infections; clinical trial; ethics, research; public health policy; Latin America; Caribbean Region. 